\title{
Modelling of a Dyeing Process and its Energy Consumption Analysis
}

\author{
By Hiroshi Ohno*, Fusao Yoshida**, Masami Morimoto**, Iori Hashimoto*** and Takeichiro Takamatus***, \\ Members, TMSJ \\ *Department of Chemical Engineering, Kobe University, Kobe
}

**Osaka Prefectural Industrial Research Institute, Enokojima, Nishi-ku, Osaka

***Department of Chemical Engineering, Kyoto University, Kyoto

Based on the Journal of the Textile Machinery Society of Japan, Transactions, Vol. 37, No. I, TI-T8 (1984-1); Vol. 38, No. I T2I-T28 (I985-I)

\begin{abstract}
The dyeing industry is a typical energy consuming industry in which a very large amount of heat and water is consumed annually. The ratio of energy cost to overall manufacturing cost in 1982, for example, was as high as $18 \%$. The dyeing industry, therefore, is one of the industries which urgently require some effective measures for saving energy. In the dyeing process, energy consumption is greatly influenced not only by the production rate, but also by the temperature of processing water, which is consumed in large volumes, as well as by counter measures for saving energy such as heat recovery. In this study, a mathematical model of dyeing process is derived from the enthalpy balance equation using the observed data. Then, the effect of water temperature and production rate on the basic unit of energy consumption is analysed using the developed mathematical model.
\end{abstract}

\section{Introduction}

The dyeing industry is a typical energy consuming industry in which a very large amount of heat and water is consumed annually. In 1982, for example, its heavy oil consumption reached 0.94 million kiloliters, corresponding to $42 \%$ of the total heavy oil consumption of the entire textile industry in Japan, i.e. 2.22 million kiloliters. The ratio of energy cost to overall manufacturing cost is as high as $18 \%$. The dyeing industry, therefore, is one of the industries which urgently require some effective measures for saving energy.

In the dyeing process, energy consumption is greatly influenced not only by the production rate, but also by the temperature of water which is consumed in a vast volume, as well as by counter measures for saving energy such as heat recovery.

This study examines such factors which influence energy consumption as (1) production rate, (2) water temperature, and (3) the amount of heat to be recovered. In order to quantitatively analyse the effect of these three factors on energy consumption in a dyeing process, mathematical model is first derived, and then, a practical approach to energy consumption analysis is demonstrated by showing how to handle and analyse actual observed data.

\section{Dyeing Process}

A schematic diagram of a dyeing process with a heat recovery system is shown in Fig. 1. In this process, it is assumed that materials are processed at a rate of $\mathrm{P}$ tons per day, and that there is no change in the weight of materials before and after processing.

The water used dyeing is water drawn directly from a river. The water is used, as it is, as cold processing water (flow rate $W_{c}\left(\mathrm{~m}^{3} / \mathrm{day}\right)$, temperature $\left.t_{c}\left({ }^{\circ} \mathrm{C}\right)\right)$, or as hot processing water $W_{h}\left(\mathrm{~m}^{3} /\right.$ day $)$ after being heated by high temperature discharge water, $t_{i}\left({ }^{\circ} \mathrm{C}\right)$, in the heat exchanger system. The steam used for this process is $Q$ (ton/day). The materials processed in this system are varied, as are the processing temperatures. The varia-

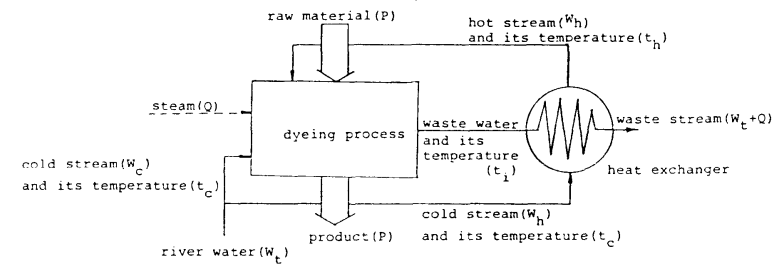

Fig. 1 Schematic diagram of dyeing process with heat exchanger unit Raw material $(P)$ Hot stream $\left(W_{h}\right)$ and its temperature $(t)$ Stream $(Q)$ Dyeing process Cold stream $\left(W_{c}\right)$ and its temperature $\left(t_{c}\right)$ Waste water and its temperature $\left(t_{i}\right)$ Waste stream $\left(W_{t}+Q\right)$ Heat exchanger Product $(P)$ River water $\left(W_{t}\right)$

tion in products as well as in the operating conditions leads to different degrees of energy consumption. Therefore, it is very important to analyse how the temperature of drawn water and the global production rate affect the total energy consumption of the process.

Used in the following analysis are observed data on daily production rate, and the amount of steam and water consumed per day.

3. Analysis of Observed Data and Mathematical Model of Energy Consumption

3.1 Daily fluctuation of observed data and pretreatment thereof

For the process without any heat recovery (called hereafter "Process 1"), 4 different kinds of data, i.e., temperature of drawn water, $t_{c}$, production rate, $P$, amount of steam consumed, $Q$, and amount of water consumed, $W_{t}$, were observed. The daily fluctuation of these values is shown in Fig. 2.

For the process with heat recovery (called hereafter "Process 2" '), a total of 5 different kinds of data, i.e., amount of hot water consumed, $W_{h}$, in addition to the above four kinds of data, were observed as shown in Fig. 3. 
Indicated also in these figures is the fluctuation in the basic unit of energy consumption (called hereafter "BUOEC"), $E=Q / P$, calculated from the data on production rate and amount of consumed steam.

As production rate as well as water temperature vary greatly, it is not an easy task to quantitatively analyse the effect of these factors on the amount of steam consumption and on BUOEC. For the same reason, it is also difficult to analyse the effect of heat recovery on economization of energy in the dyeing process.

First, in order to find out qualitative relations among these variables, a pretreatment of the observed data is performed, and then several fundamental statistics are calculated.

The dyeing process from which the observed data are obatined is operated $8 \mathrm{hr} /$ day on week days, Monday through Friday, for a half-day on Saturdays, and is closed on Sundays. In Figs. 2 and 3 , Sundays do not appear on the abscissa.

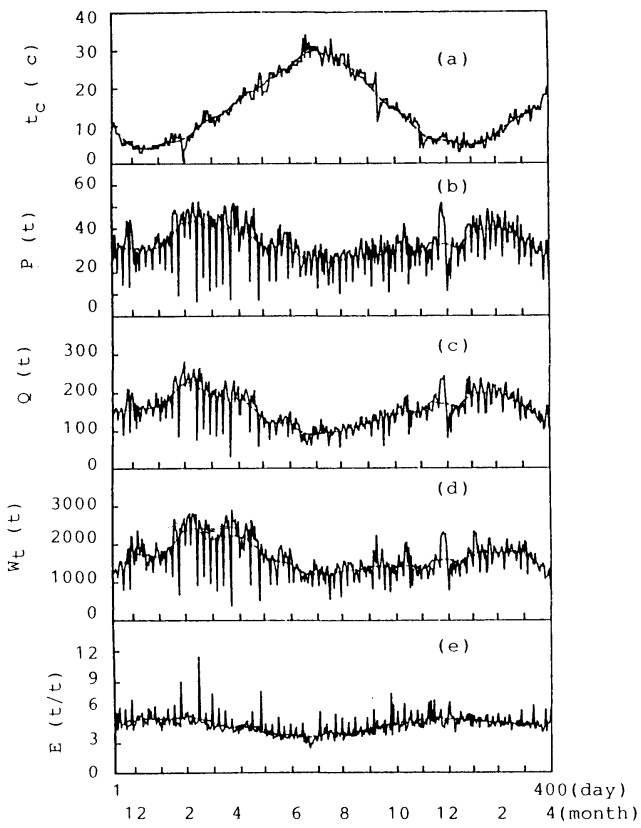

Fig. 2 Measured data and their moving averages (Non-heat-recovery process)

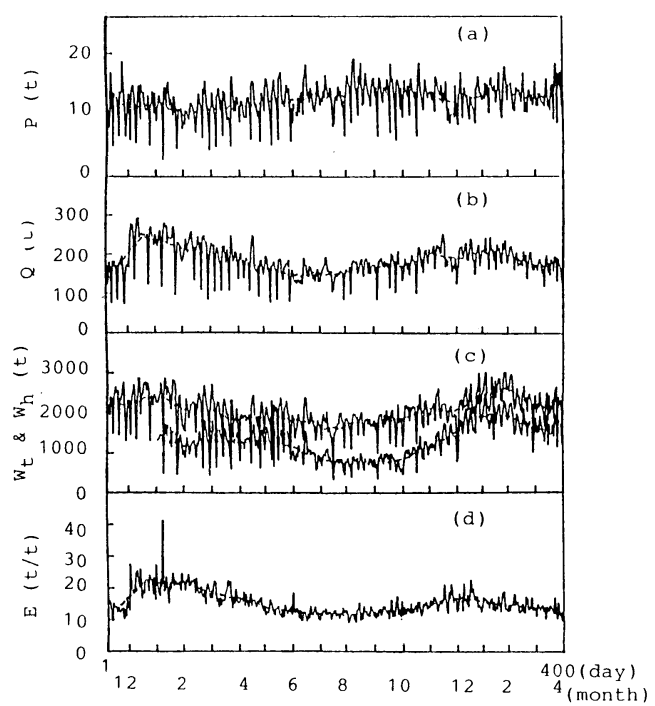

Fig. 3 Measured data and their moving average (Heat-recovery process)

All data except water temperature show a cyclic variation of 6 days. This cycle is disturbed during summer and new year holi- days. In order to eliminate this cyclic variation as well as high frequency noise, a moving average over a 24 day period is calculated for each datum. The results are smooth curves shown in Figs. 2 and 3.

The moving averages of $P, Q$ and $W_{t}$ display the same tendency, and therefore suggest strong correlations among these variables.

On the other hand, the correlation between the water temperature, $t_{c}$, and the amount of consumed steam, $Q$, is obscure.

Means, standard deviations and coefficients of variation of each variable are shown in Table 1. The matrix of correlation co-

Table 1 Statistic characteristics of data

(a) Non-heat-recovery process

\begin{tabular}{c|c|c|c}
\hline \hline & $m$ & $\sigma$ & $C_{v}$ \\
\hline$P$ & 32.72 & 9.44 & 29 \\
\hline$Q$ & 154.8 & 46.8 & 30 \\
\hline$W_{t}$ & 1644 & 486 & 29 \\
\hline$t$ & 14.0 & 8.6 & 60 \\
\hline
\end{tabular}

Number of observation $=400$

(b) Heat-recovery process

\begin{tabular}{c|c|c|c}
\hline \hline & $m$ & $\sigma$ & $C_{v}$ \\
\hline$P$ & 12.41 & 2.70 & 22 \\
\hline$Q$ & 183.6 & 39.9 & 22 \\
\hline$W_{t}$ & 2046 & 429 & 21 \\
\hline$W_{h}$ & 1130 & 573 & 51 \\
\hline
\end{tabular}

Number of observation $=400$

Table 2 Correlation table

2) Non-heat-recovery process

\begin{tabular}{c|c|c|c|c}
\hline \hline & $P$ & $Q$ & $W_{t}$ & $t_{c}$ \\
\hline$P$ & 1 & 0.872 & 0.886 & -0.290 \\
\hline$Q$ & 0.872 & 1 & 0.849 & -0.624 \\
\hline$W_{t}$ & 0.886 & 0.849 & 1 & -0.317 \\
\hline$t_{c}$ & -0.290 & -0.624 & -0.317 & 1 \\
\hline
\end{tabular}

(b) Heat-recovery process

\begin{tabular}{c|c|c|c|c}
\hline \hline & $P$ & $Q$ & $W_{t}$ & $t_{c}$ \\
\hline$P$ & 1 & 0.445 & 0.537 & 0.125 \\
\hline$Q$ & 0.445 & 1 & 0.783 & -0.533 \\
\hline$W_{t}$ & 0.534 & 0.783 & 1 & -0.501 \\
\hline$t_{c}$ & 0.125 & -0.533 & -0.501 & 1 \\
\hline
\end{tabular}

efficients, which is a useful index for expressing the strength of relationships between variables, is also shown in Table 2 . As is clear from Table 2 , the correlations among $P, Q$ and $W_{t}$ are very strong, while those between water temperature, $t_{c}$, and the other variables seem to be quite small.

For example, the amount of consumed steam, $Q$, has a strong relationship with production rate, $P$. The variation of $Q$ resulting from the variation of $P$ is so large that the effect of the fluctuation of water temperature on the variation of $Q$ might be obscured.

In such cases, an analysis based on the concept of partial correlation is very useful. In other words, by eliminating the effect of 
the variations of other variables on two specified variables, the relationship between these two variables can be revealed by the partial correlation coefficient, the calculated values of which are shown in Table 3.

Table 3 Partial correlation

\begin{tabular}{c|c|c|c}
\hline \hline \multirow{2}{*}{$\begin{array}{c}\text { Non-correlated } \\
\text { variables }\end{array}$} & \multirow{2}{*}{$\begin{array}{c}\text { Correlated } \\
\text { variables }\end{array}$} & \multicolumn{2}{|c}{ Partial correlation } \\
\cline { 3 - 4 } & $\begin{array}{c}\text { Non-heat- } \\
\text { recovery }\end{array}$ & Heat-recovery \\
\hline$P$ & $Q, t$ & -0.792 & -0.662 \\
\hline$t_{c}$ & $Q, P$ & 0.924 & 0.610 \\
\hline$P$ & $W, t_{\bullet}$ & -0.135 & -0.678 \\
\hline$t_{t}$ & $W_{,}, P$ & 0.875 & 0.695 \\
\hline
\end{tabular}

The partial correlation coefficient between $Q$ and $t$ after eliminating the influence of $P$ is -0.792 , the absolute value of which is greater than that of the coefficient of correlation given in Table 2 i.e. -0.624 . This means that the amount of steam consumed, $Q$, is strongly affected by the water temperature, $t$.

The partial correlation coefficient between $W$, and $P$ is quite large, while the coefficient of correlation between these two variables is also large. On the other hand, the partial correlation coefficient between $W_{t}$ and $t_{r}$ is much smaller than the coefficient of correlation between these two variables. This means that the amount of consumed water, $W_{t}$, is determined mainly by the production rate $P$, and almost irrespective of water temperature $t_{c}$.

\subsection{Mathematical model for energy consumption}

In order to express quantitatively the relationships among the variables, the observed values of which are given in Figs. 2 and 3 , a mathematical model is derived based on the enthalpy balance equation. In the process shown in Fmig. 1, $H_{p}, H_{q}, H_{c}$, and $\boldsymbol{H}_{h}$ denote enthalpies brought into the process along with raw materials, steam, cold processing water, and hot processing water, respectively. Also $H_{p}{ }^{\prime}$, and $H_{w}$ denote the enthalpies brought away by products and discharged hot water. Thus, the enthalpy balance is given by

$$
H_{p}+H_{q}+H_{c}+H_{t}=H_{h}=H_{P}{ }^{\prime}+H_{w}+Q_{0}
$$

where $Q_{0}$ is the overall heat loss which is inherent in a dyeing process irrespective of production rate. The units used in Eq. (1) are $\mathrm{kcal} /$ day. Assuming that the amount of enthalpy needed for dyeing is proportional to production rate, the following equation holds:

$$
H_{P}{ }^{\prime}-H_{P}=a P
$$

By substituting Eq. (2) into Eq. (1), and setting the base of enthalpy at $0^{\circ} \mathrm{C}$, we have

$$
h Q+W_{c} t_{c}+W_{h} t_{h}=Q_{0}^{\prime}+a^{\prime} P+\left(W_{c}+W_{h}+Q\right) t_{i}
$$

where $h(\mathrm{kcal} / \mathrm{kg})$ is the enthalpy of $1 \mathrm{~kg}$ of stream. The specific heat of water is assumed to be $1\left(\mathrm{kcal} / \mathrm{kg}^{\circ} \mathrm{C}\right)$, irrespective of water temperature, and moreover

$$
Q_{1}^{\prime}=10^{-3} \cdot Q_{11} \text {, and } a^{\prime}=10^{-3} \cdot a
$$

It is also assumed that the flow rate of discharged hot water is equal to the sum of the amount of water consumed, $W_{t}$, and the amount of steam consumed, $Q$, and that the temperature of discharged hot water is $t_{i} . t_{h}$ in Eq. (3) is the temperature of hot processing water, and $t_{h}$ is equal to the temperature of cold processing water, $t_{c}$, in a process without any heat recovery system. In a process with a heat recovery system, the temperature of hot processing water, $t_{h}$, is given by the following equation, derived using both an enthalpy balance around the heat exchanger system and the heat transfer rate equation (see Appendix):

$$
t_{h}=r t_{c}+(1-r) t_{i}
$$

where $r=1$ for a process without any heat recovery system.

By substituting Eq. (4) into Eq. (3), the following equation is derived:

$$
\left.Q=\frac{1}{h-t_{\mathrm{i}}}\left\{Q_{0}{ }^{\prime}+a^{\prime} P+W_{c}+r W_{h}\right)\left(t_{i}-t_{c}\right)\right\} \cdots(5)
$$

Equation (5) gives a mathematical model expressing the energy consumption in a dyeing process, (this model is referred to as Model- $Q_{r}$ hereafter).

For a process without any heat recovery system, it holds that $r=1$, and $W_{t}=W_{c}+W_{h}$. Therefore, the mathematical model to express the energy consumption in this process is given by

$$
Q=\frac{1}{h-t_{i}}\left\{Q_{0}{ }^{\prime}+a^{\prime} P+{ }_{t}\left(t_{i}-t_{c}\right)\right\} \cdots \cdots \cdots \cdots \cdots(6)
$$

This model is subsequently referred to as Model- $Q_{1}$.

The unknown parameters in Eq. (5) are $Q_{0}{ }^{\prime}, a^{\prime}, h, t_{i}$ and $r$.

In order to estimate these unknown parameters from the observed data, Eq. (5) is rewritten as follows,

$$
\begin{aligned}
Q & =\left(\frac{Q_{0}^{\prime}}{h-t_{i}}\right)+\left(\frac{a^{\prime}}{h-t_{i}}\right) P+\left(\frac{t_{i}}{h-t_{i}}\right)\left(W_{c}+r W_{h}\right) \\
& -\left(\frac{1}{h-t_{i}}\right)\left(W_{c}+r W_{h}\right) t_{c} \quad \ldots \ldots \ldots \ldots \ldots \ldots \ldots \ldots \ldots \ldots \ldots \ldots \ldots \ldots \ldots
\end{aligned}
$$

The right side of Eq. (7) is a linear function with respect to the coefficients $\left(Q_{0}{ }^{\prime} / h-t_{i}\right),\left(a^{\prime} / h-t_{i}\right),\left(t_{i} / h-t_{i}\right)$ and $\left(-1 / h-t_{i}\right)$. If a

Table 4 Estimated model parameter

\begin{tabular}{c|c|c|c|c|c|c|c}
\hline \hline Model & $r$ & $\begin{array}{c}Q_{0} \\
(\mathrm{kcal} / \mathrm{day})\end{array}$ & $\begin{array}{c}a \\
(\mathrm{kcal} / \mathrm{t})\end{array}$ & $\begin{array}{c}t ; \\
\left({ }^{\circ} \mathrm{C}\right)\end{array}$ & $\begin{array}{c}h \\
(\mathrm{kcal} / \mathrm{kg})\end{array}$ & $\begin{array}{c}R \\
(-)\end{array}$ & $\begin{array}{c}\varepsilon \\
(-)\end{array}$ \\
\hline$Q_{1}$ & 1 & $18.9 \times 10^{6}$ & $1.86 \times 10^{6}$ & 29.3 & 717 & 0.9637 & 12.5 \\
\hline$Q_{r}$ & 0.436 & $24.2 \times 10^{6}$ & $1.88 \times 10^{6}$ & $39 *, 59^{* *}$ & 639 & 0.7695 & 17.3 \\
\hline
\end{tabular}

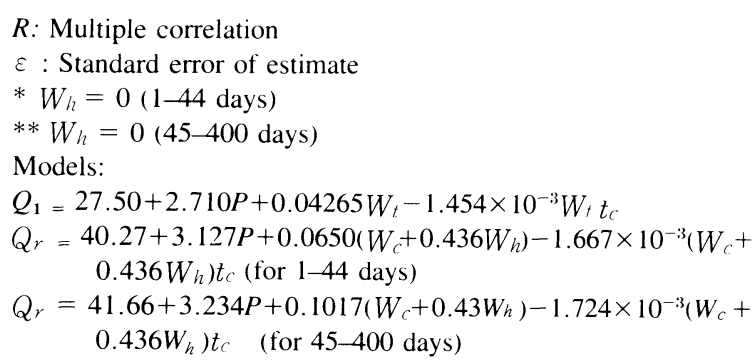


value is assumed for $r$, these coefficients can be calculated using the linear least square method. By varying $r$ and repeating this fitting, the mean squared deviation of $Q$ from the observed data can be minimized. This minimum gives the best estimates of coefficients and thus also the parameters.

Here, unknown parameters are determined by using repeatedly the least square method for various assumed values of $r$. The estimated values of unknown parameters, $Q_{0}, a, r, h$ and $t_{i}$, as well as the mathematical model equations obtained in terms of these parameter values are shown in Table 4 . The change with time of the amount of stream consumed, $Q$, which is calculated by using the estimated parameter values, is shown in Figs. 4(a) and (b). The scatter diagram between the observed data and the

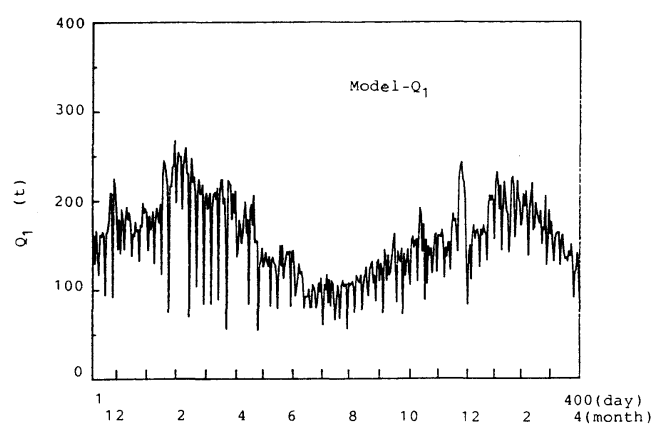

(a) A process without any heat recovery system

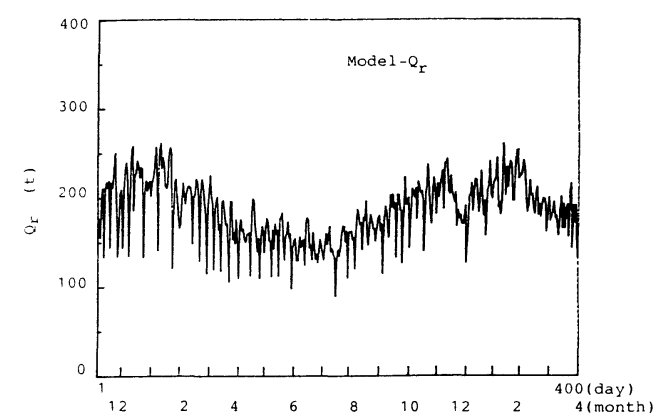

(b) A process with a heat recovery system

Fig. 4 Calculated vales of $Q$ (Using Model- $Q_{1}$ and Model-Qr)

estimated values of $Q$ is shown in Figs. 5(a) and (b). From these figures, it is safe to conclude that the mathematical models derived here are fairly accurate.

\section{Analysis of Energy Consumption for No Heat Recovery System}

The amount of energy consumed per unit product, $E(=Q / P)$, is a useful index for analysing the state of energy utilization.

From eq. (6), we have

$$
E=\frac{1}{P\left(h-t_{i}\right)}\left\{Q_{0}{ }^{\prime}+a^{\prime} P+\left(t_{i}-t_{c}\right) W_{t}\right\}
$$

Judging from the correlation coefficients shown in Tables 2 and $3, W_{t}$ has only a weak correlation with $t_{c}$, but a very strong correlation with $P$. Accordingly, the relationship between $W_{t}$ and $P$ may be assumed to be as follows:

$$
W_{t}=W_{1}+b P
$$

In other words, it is assumed that the amount of water constmed is expressed, regardless of the production rate, by the sum of both a term proportional to the production rate and a constant
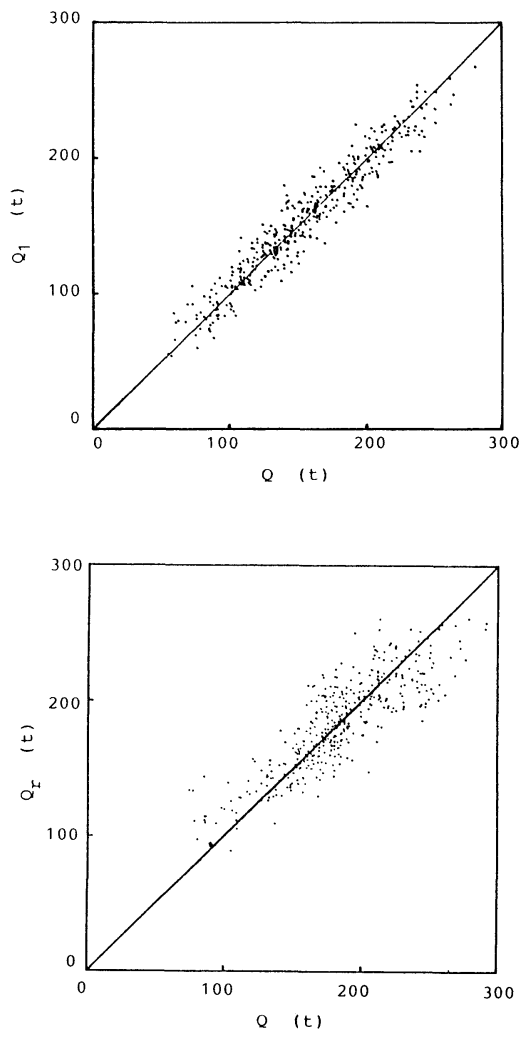

Fig. 5 Scatter diagram between $Q$ observed and $Q_{\mathrm{r}}$ calculated from the models developed

term which is related to the amount of water used. The values estimated from the observed data are: $W_{0}=155.4$ and $b=45.5$, and the correlation coefficient $R$ is 0.8861 .

By substituting Eq. (9) into Eq. (8), the following equation is obtained:

$$
E=\frac{1}{h-t_{i}}\left\{\frac{Q_{0}{ }^{\prime}}{P}+a^{\prime}+t_{i}\left(\frac{W_{0}}{\mathrm{P}}+\mathrm{b}\right) \mathrm{t}_{\mathrm{c}}\left(\frac{W_{0}}{P}+b\right)\right\} \cdots \cdots(10)
$$

As is clear from Eq. (10), $E$ is related only to variables $P$ and $t_{c}$.

\subsection{Effect of water temperature on BUOEC}

In order to analyse the effect of water temperature on BUOEC, the relationship between $t_{c}$ and $E$ when $P$ changes is first derived as shown in Fig. 6.

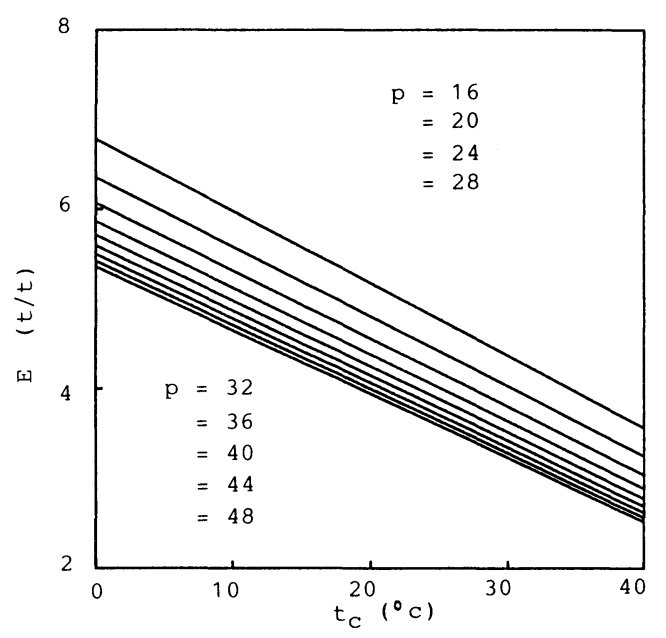

Fig. 6 Relationship between BUOEC, $E$, and water temperature, $t_{c}$

When $P$ is constant, the change in BUOEC caused by a change in the water temperature of $1^{\circ} \mathrm{C}, \Delta E_{t}$, can be obtained from Eq. 
(10) as follows:

$$
\Delta E_{t}=\left(\frac{\partial E}{\partial t_{c}}\right)_{P}=-\frac{0.4462}{P}-0.6315
$$

As an index for expressing the effect of water temperature, $K_{t m}$, is defined as

$$
K_{t m}=\left(\Delta E_{t m} / E_{m}\right) \times 100
$$

where $E_{m}$ and $\Delta E_{t m}$ express the values obtained from Eqs. (10) and (11), respectively, with the mean values of $P$ and $t_{c}$ being used.

The calculated value of $K_{t m}$ for the mean values shown in Table 1(a) is -1.60 . As is clear from Eq. (11), as the production rate decreases, the absolute value of $\Delta E_{t}$ increases. However, the value of $E$ itself also increases, though the index of the effect of water temperature, $K_{t m}$, remains almost constant. In other words, BUOEC decrease by $1.6 \%$ for $1{ }^{\circ} \mathrm{C}$ increase in the water temperature, even if the production rate fluctuates.

\subsection{Effect of production rate on BUOEC}

The relationship between $P$ and $E$ is shown in Fig. 7 with $t_{c}$ as a parameter. When $t_{c}$ is constant, the change in BUOEC result-

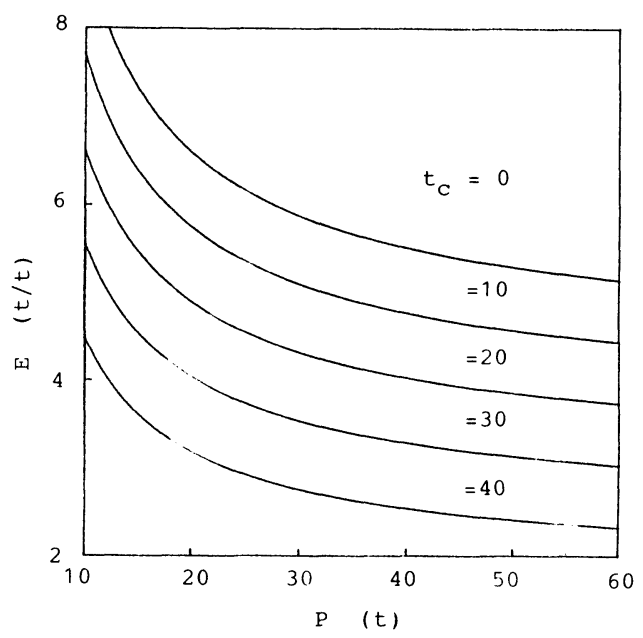

Fig. 7 Relationship between BUOEC, $E$ and production rate, $P$

ing from a change in production rate of 1 ton, $\Delta E_{p}$, is given by:

$$
\Delta E_{P}=\left(\frac{\partial E}{\partial P}\right)_{t_{c}}=\frac{1}{P^{2}}\left(0.4462 t_{c}-43.72\right) \cdots
$$

Similarly, the index $K_{p m}$ is introduced to express the effect of variation in the production rate on BUOEC.

$$
K_{p m}=\left(\Delta E_{p m} / E_{m}\right) \times 100 \quad(\% / \mathrm{t})
$$

The value of $K_{p m}$, calculated using the values shown in Table $1(\mathrm{a})$, is -0.70 .

This means that BUOEC decreases by $0.7 \%$ as the production rate increased by 1 ton. As is clear from both Eq. (13) and Fig. 7 the sensitivity of BUOEC to the change in the production rate, i.e., $\Delta E_{p}$, is only slightly influenced by the change in water temperature, but is greatly influenced by the actual value of the production rate $P$ itself. That is, as $P$ becomes smaller, the influence of changes in the production rate becomes larger.

\section{Analysis of Energy Consumption for Heat Recovery System}

5.1 Effect of heat recovery on BUOEC

From Eq. (5) BUOEC in this process is expressed as follows:

$$
E=\frac{1}{P\left(h-t_{i}\right)}\left\{Q_{0}^{\prime}+a^{\prime} P+\left(t_{i}-t_{c}\right)\left(W_{c}+r W_{h}\right)\right\}
$$

Denoting BUOEC in a process without any heat recovery system by $\bar{E}$ (which can be obtained from Eq. (15) by making $r=1$ ), the reduction in BUOEC resulting from the installation of a heat recovery system is given by

$$
\frac{\bar{E}-E}{\bar{E}}=\frac{(1-r) W_{h}\left(t_{i}-t_{c}\right)}{Q_{0}^{\prime}+a^{\prime} P+\left(t_{i}-t_{c}\right) W_{t}}
$$

The amount of the decrease in BUOEC depends on production rate, water temperature and the amount of water consumed, which is the linear function of production rate $P$ :

$$
W_{t}=W_{c}+W_{h}=W_{0}+b P=968.4+83.47 P
$$

By substituting Eq. (17) into Eq. (16) and using the estimated parameter values, Eq. (16) is expressed as follows:

$$
\frac{\bar{E}-E}{\bar{E}}=\frac{0.5639(968.4+83.47 P)\left(t_{i}-t_{c}\right) \alpha}{24160+1878 P+(968.4+83.47 P)\left(t_{i}-t_{c}\right)}
$$

As is clear from Eq. (18), the amount of the decrease in BUOEC, $1-E / \bar{E}$, is proportional to the term $\left(\alpha=W_{h} / W_{t}\right)$ which expresses the ratio of the amount of water being heated in the heat exchange system to the total amount of water consumed. Therefore, the relationship between $(\bar{E}-E / \bar{E}) /\left(W_{h} / W_{t}\right)$ and $\left(t_{i}-t_{c}\right)$ is shown in Figure 8, in which the effect of heat recovery on

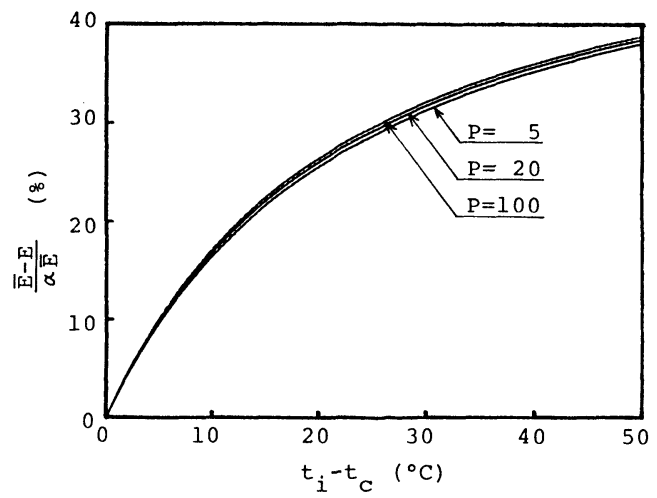

Fig. 8 Reduction in BUOEC resulting from the installation of a heat recovery system

BUOEC is shown, as expected, more significant when the water temperature is low in winter time. For example, when $t_{i}-t_{c}=$ $40^{\circ} \mathrm{C}$, the decrease in BUOEC is expected to be as much as $36 \%$. On the other hand, the effect of heat recovery is almost constant even though the production rate increases.

\subsection{Effect of wâter temperature on BUOEC}

The sensitivity of BUOEC with respect to water temperature $t_{c}$ is expressed by

$$
\frac{\partial E}{\partial t_{c}}=\frac{-1}{P\left(h-t_{i}\right)}\left(W_{c}+r W_{h}\right)
$$

For a process without any heat recovery system, the following equation is obtained.

$$
\frac{\partial \bar{E}}{\partial t_{c}}=\frac{-1}{P\left(h-t_{i}\right)}\left(W_{t}\right)
$$

Therefore, the ratio of the above two sensitivities is a linear function of $\left(W_{h} / W_{t}\right)$ as follows:

$$
\frac{\partial E}{\partial t_{c}} / \frac{\partial \bar{E}}{\partial t_{c}}=1-\alpha(1-r)
$$

Equation (19) takes the minimum value of $r=0.4361$ when $W_{h} / W_{t}=1$. In other words, if all of the consumed water is preheated in a heat recovery system, then the undesirable effect of the variation of water temperature on BUOEC can be reduced 
almost to $1 / 2$.

As discussed above, the heat recovery system plays an important role not only in decreasing BUOEC, but also in alleviating the bad influence of water temperature variation.

\subsection{Effect of production rate on BUOEC}

The following is an analysis of how the sensitivity of BUOEC to the change in the production rate is influenced by the existence of a heat recovery system.

By rewriting Eq. (15), we have:

$$
E=\frac{1}{P\left(h-t_{i}\right)}\left\{Q_{0}^{\prime}+a^{\prime} P+\left(t_{i}-t_{c}\right) W_{t}(1-\alpha(1-r))\right\}
$$

By substituting Eq. (17) into the above equation, and taking the partial derivative of $E$ with respect to $P$, the following expression is obtained:

where $W_{0}$ is 968.4 .

$$
\frac{\partial E}{\partial P}=\frac{-1}{P^{2}\left(h-t_{i}\right)}\left\{Q_{0}^{\prime}+W_{0}\left(t_{i}-t_{c}\right)(1-\alpha(1-r))\right\}
$$

For a process without any heat recovery system, the following equation is obtained:

$$
\frac{\partial \bar{E}}{\partial P}=\frac{-1}{P^{2}\left(h-t_{i}\right)}\left\{Q_{0}^{\prime}+W_{0}\left(t_{i}-t_{c}\right)\right\}
$$

The value of $E / \partial P$ is negative. Thus, the sensitivity of BUOEC with respect to production rate $P$ decreases as $P$ increases. The absolute value of the sensitivity is smaller when a heat recovery system is implemented in a dyeing process. This is shown by

$$
\frac{\partial E / \partial P}{\partial \bar{E} / \partial P}=\frac{Q_{0}^{\prime}+W_{0}\left(t_{i}-t_{c}\right)\{1-(1-r) \alpha\}}{Q_{0}^{\prime}+W_{0}\left(t_{i}-t_{c}\right)}<1 \quad \cdots(22)
$$

Thus, the rate of decrease in the effect of production rate on BUOEC resulting from the installation of a heat recovery system is determined by the temperature difference $\left(t_{i}-t_{c}\right)$ as well as by the term $\alpha=\left(W_{h} / W_{t}\right)$. This relationship is shown in Figure 9.

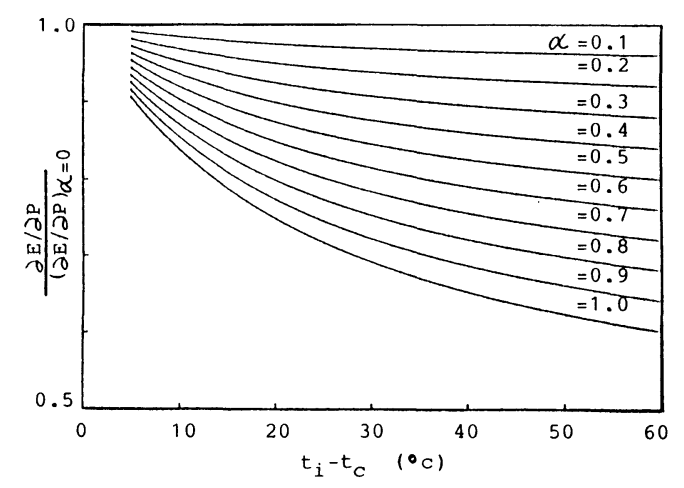

Fig. 9 Effect of the installation of a heat recovery system on the sensitivity of BUOEC to production rate

\section{Conclusion}

In a process such as the dyeing process, in which vast water is consumed and its fairly large proportion is heated, the water temperature greatly affects BUOEC as dose the production rate. In this study, a mathematical model of the dyeing process is derived from the enthalpy balance equation.

Using this mathematical model, the importance of the effect of water temperature and production rate on BUOEC is analysed. The accuracy of this model is checked by comparing real data with values calculated from the model, and the following results are derived.
(1) For a process without any heat recovery system, a $1{ }^{\circ} \mathrm{C}$ increase in the water temperature results in an increase of $1.6 \%$ in BUOEC, and a 1 ton decrease in the production rate results in an increase of $0.7 \%$.

(2) For a process with a heat recovery system, the effect of heat recovery on BUOEC is greatly affected by water temperature. In the winter period when the water temperature is very low, the reduction in BUOEC is expected to be as much as $36 \%$. The effect of heat recovery does not change even though production rate increases. The effect of the variation of water temperature on BUOEC is reduced by as much as $56 \%$ by installing a heat recovery system.

Thus, a heat recovery system in a dyeing process plays an important role in reducing the effect of water temperature and production rate on BUOEC.

\section{$\ll$ APPENDIX 》 \\ Input and Output Relation in a Heat Exchanger}

In the general heat exchanger shown in Fig. $\mathrm{A}$, let $W, C_{p}$, $T_{i}$, and $T_{0}$ be flow rate, specific heat, inlet and outlet temperature of the hot stream, respectively. Let $w, c_{p}, t_{i}$, and $t_{0}$ be those of cold stream, respectively.

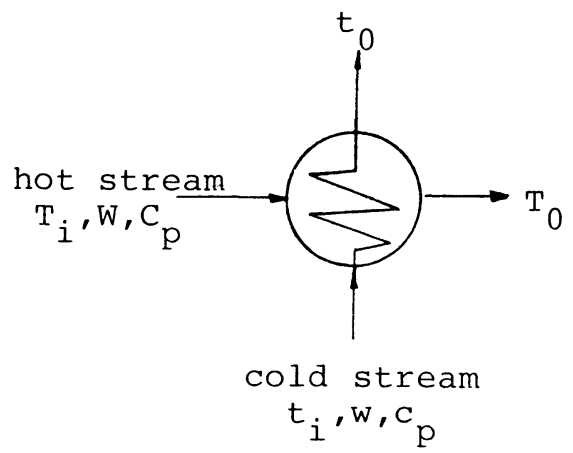

Fig. A Heat exchanger unit

The heat balance and the heat transfer rate equations are given as follows:

$$
\begin{aligned}
& W C_{\rho}\left(T_{i}-T_{0}\right)=w c_{\rho}\left(t_{0}-t_{i}\right) \cdots \cdots \cdots \cdots \cdots \cdots \cdots(\mathrm{A}-1) \\
& W C_{p}\left(T_{i}-T_{0}\right)=A U \cdot \Delta t \quad \cdots \cdots \cdots \cdots \cdots \cdots \cdots \cdots \cdots \cdots(\mathrm{A}-2)
\end{aligned}
$$

where $A$ and $U$ are the heat transfer area and the overall heat transfer coefficient, respectively.

$\Delta t$ is a logarithmic mean temperature difference, defined by:

$$
\Delta t=\left(\Delta t_{2}-\Delta t_{1}\right) / \log \left(\Delta t_{2} / \Delta t_{1}\right) \cdots \cdots \cdots \cdots \cdots(\mathrm{A}-3)
$$

where $\Delta t_{2}$ and $\Delta t_{1}$ are the difference of stream temperatures at the inlet and outlet of the heat exchanger. For a countercurrent heat exchanger, $\Delta t_{2}$ and $\Delta t_{1}$ are given by the following equations:

$$
\Delta t_{2}=T_{i}-t_{0}, \Delta t_{1}=T_{0}-t_{i} \cdots \cdots \cdots \cdots \cdots \cdots \cdots \cdots \cdots \cdots \cdots(\mathrm{A}-4)
$$

By rewriting the right hand side of Eq. (A-2), we have:

$$
\begin{aligned}
W C_{\rho}\left(T_{i}-T_{0}\right) & =A U \cdot \Delta t \\
& =A U\left(T_{i}-t_{0}-T_{0}+t_{i}\right) / \log \left(\Delta t_{2} / \Delta t_{1}\right) \\
& =A U\left(T_{i}-T_{0}\right)\left(1-\frac{W C_{\rho}}{w c_{\rho}}\right) / \log \left(\frac{\Delta t_{2}}{\Delta t_{1}}\right)
\end{aligned}
$$

From the above equation, the following equations can be obtained:

$$
\log \frac{\Delta t_{2}}{\Delta t_{1}}=\left(\frac{A U}{W C_{p}}\right)\left(1-\frac{W C_{p}}{w c_{p}}\right) \cdots \cdots \cdots \cdots \cdots(\mathrm{A}-5)_{1}
$$

or

$$
\frac{T_{i}-t_{0}}{T_{0}-t_{i}}=\exp \left\{\frac{A U}{W C_{p}}\left(1-\frac{W C_{p}}{w c_{p}}\right)\right\} \quad \cdots \cdots \cdots(\mathrm{A}-5)_{2}
$$


where it is assumed that $W C_{p} \neq w c_{p}$.

By rearranging Eqs. (A-1) and (A-5) $)_{2}$, the following equations are obtained:

$$
\begin{aligned}
& T_{0}-t_{i}=\exp \left\{\frac{A U}{W C_{p}}\left(\frac{W C_{p}}{w c_{p}}-1\right)\right\}\left(T_{i}-t_{0}\right) \\
& T_{i}-T_{0}=\left(\frac{w c_{p}}{W C_{p}}\right)\left(t_{0}-t_{i}\right)
\end{aligned}
$$

By adding both sides of the above two equations,

$$
T_{i}-t_{i}=C_{1}\left(T_{i}-t_{0}\right)+C_{2}\left(t_{0}-t_{i}\right)
$$

where

$$
C_{1}=\exp \left\{\frac{A U}{W C_{\rho}}\left(\frac{W C_{\rho}}{w c_{\rho}}-1\right)\right\}, \quad C_{2}=\frac{w c_{\rho}}{W C_{p}}
$$

From the above equation, we have

$$
t_{0}=\left(\frac{1-C_{1}}{C_{2}-C_{1}}\right) T_{i}+\left(\frac{C_{2}-1}{C_{2}-C_{1}}\right) t_{i}
$$

If the coefficient of $t_{i}$ is expressed by $r$, then the coefficient of $T_{i}$ is $(1-r)$.

Therefore, the outlet temperature of the cold stream, $t_{0}$, is expressed by the following linear function of the inlet tempera- tures of the cold and hot streams, $T_{i}$ and $t_{i}$.

$$
t_{0}=(1-r) T_{i}+r t_{i}
$$

Even for a concurrent heat exchanger, the outlet temperature of the cold stream can also be expressed by a linear function of the inlet temperatures of the cold and hot streams, if the temperature differences of both stream at the inlet and outlet of the heat exchanger are expressed by $\Delta t_{2}=T_{1}-t_{i}, \quad \Delta t_{i}=T_{0}-t_{0}$

By applying Eq. (A-6) to the heat exchanger shown in Fig. 1, Eq. (4) in the text can be obtained.

$$
t_{h}=r t_{c}+(1-r) t_{i}
$$

where

$$
\begin{gathered}
r=\frac{C_{2}-1}{C_{2}-C_{1}}, C_{1}=\exp \left\{A U\left(\frac{1}{W_{h}}-\frac{1}{Q+W_{t}}\right)\right\} \\
C_{2}=\frac{W_{h}}{Q+W_{t}}
\end{gathered}
$$

Although the parameter $r$ in Eq. (4) is a function of $Q, W_{h}$ and $W_{t}$ as shown in the above, $r$ is assumed to be constant in this paper.

In case that $W C_{p}=w c_{p} \quad \Delta t_{1}=\Delta t_{2}$ holds, $r$ is given by:

$$
r=1 /\left\{1+A U /\left(Q+W_{t}\right)\right\}
$$

